\title{
Integrated energy service demand evaluation based on AHP and entropy weight method
}

\author{
Xianjun $\mathrm{Qi}^{1,2}$, Mucong Zhou ${ }^{2 *}$ \\ ${ }^{1}$ Anhui Provincial Laboratory of Renewable Energy Utilization and Energy Saving, Hefei University of Technology, Hefei, Anhui Province, \\ 230009, China \\ ${ }^{2}$ School of Electrical and Automatic Engineering, Hefei University of Technology, Hefei, Anhui Province, 230009, China
}

\begin{abstract}
In the context of the energy revolution, integrated energy services have ushered in a period of opportunities for rapid development. To assess the demand of users of integrated energy services, we establish indexes of demand of integrated energy service users. We got the assessment model based on the analytic hierarchy process (AHP) and entropy weight method. The proposed assessment model is tested on 50 users, and $K$-means clustering algorithm is used to cluster users. The characteristics of the service requirements of each type are analysed.
\end{abstract}

\section{Introduction}

In recent years, with the accelerated reform of electric power system and the rapid development of technology, profound changes in energy system structure have been triggered ${ }^{[1]}$. In the context of the energy revolution, integrated energy services have ushered in a period of opportunities for rapid development ${ }^{[2]}$. Integrated energy service is a service mode combining four types of services: energy basic service, distributed energy service, energy conservation and emission reduction, and demand response service. The development of integrated energy services will play a substantial role in improving the efficiency of the energy system. The integrated energy service market is huge and has a broad development prospect, which has attracted extensive attention and discussion in recent years ${ }^{[3]}$.

From the successful practice of the United States, Japan, Germany, etc, integrated energy service is not only the promotion of emerging energy technologies, such as integrated energy system, renewable energy, smart grid, but also the reconstruction of energy consumption and supply structure ${ }^{[4]}$. Therefore, the key to realizing the scale development of integrated energy service is to build mature market promotion maximums and operation technologies. At present, research on integrated energy services mainly aim at the economic and environmental assessment $^{[5]}$, market size analysis ${ }^{[6]}$, trading and game methods and experience introduction ${ }^{[7]}$. When carrying out integrated energy services, the assessment of integrated energy services requirement is conducive to integrated energy service companies to accurately grasp energy use characteristics of users and provide users with accurate integrated energy services, which can not only improve service efficiency, but also save resources and costs.

With the background of integrated energy service, this paper establishes indexes of demand of integrated energy service users. Based on the AHP and entropy weight method, get the assessment model. The demand of 50 users is assessed. $K$-means clustering algorithm is used to cluster users. The characteristics of the service requirements of each type are analysed.

The main contributions of this paper:

1) this paper establishes the index systems to reflect the demand of users of integrated energy service.

2) The index is weighted by the AHP and entropy weight method to get the assessment model

3) use $K$-means clustering algorithm to cluster users, which can help to analyse the characteristics of demand of users

\section{Demand Indexes of Integrated Energy Service}

In this paper, the comprehensive evaluation system is divided into three levels: first-level assessment index, second-level assessment index and the final integrated assessment. The first level index is the category of the second level index and does not have quantitative data itself.

*Corresponding author's e-mail: 1253931981@qq.com 


\subsection{Integrated energy service demand assessment indicator}

According to the three types of integrated energy service, the first-level indexes are divided into basic energy service demand, distributed energy service demand, energy conservation and emission reduction demand, demand response service demand and subjective factors. The firstlevel indexes are shown in Figure 1. the second-level indexes are shown in Figure 2.

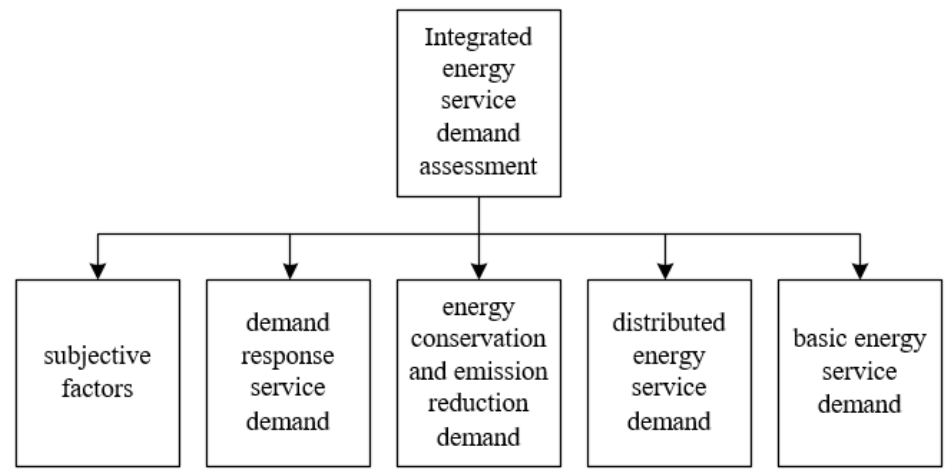

Figure 1. First-level indexes
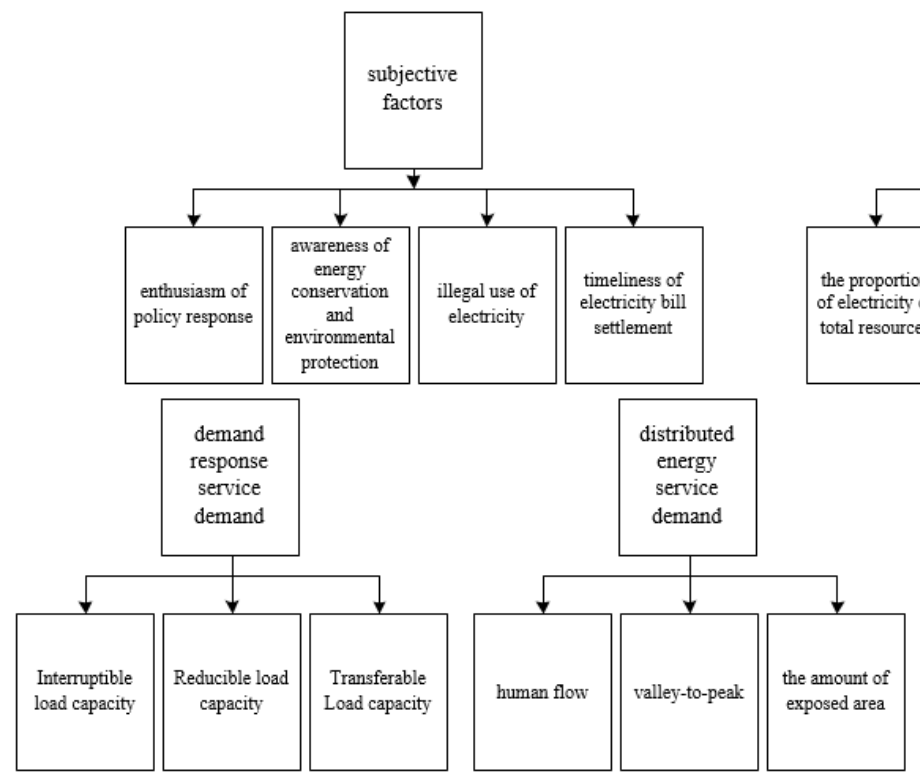

Figure 2.Second-level indexes
The indicators of basic energy service demand include the number of electric vehicles, thermal energy demand, energy costs and energy efficiency. The number of electric vehicles refers to the total number of electric vehicles contained by users. Charging electric vehicles during the trough of electricity price is also one of the businesses of energy basic services. Users can also entrust comprehensive energy service companies to build charging piles for electric vehicles, so the number of electric vehicles is chosen as a secondary indicator. Heat storage is also one of the energy infrastructure services during the trough of electricity prices. Thermal energy demand is used to measure the demand of thermal for this user. The unit of indicator is kJ. The energy costs can reflect the importance of energy infrastructure services to users. Energy efficiency refers to the ratio between the effective use of energy and the actual consumption of energy. It is an indicator reflecting the level of energy consumption and utilization effect, namely the degree of energy efficiency utilization. And it can reflect the demand for intelligent energy management services.

The indicators of distributed energy service demand include the amount of exposed area, valley-to-peak and human flow. If the amount of exposed area of a building is large, photovoltaic power generation can be given priority consideration. The amount of exposed area of a building can reflect the demand for distributed energy services. Customers with a large valley-to-peak may have a large energy storage demand, so peak-valley difference is also a second-level indicator. Places with large human flow have higher quality requirements for cold, heat and electricity, and there is a certain demand for the co-supply system of cool, heat and power, so the human flow is selected as an indicator.

The indicators of energy conservation and emission reduction demand include high energy consumption 
equipment capacity, waste heat and pressure resources, carbon emissions and the proportion of electricity of total resources. If the capacity of energy-consuming equipment is high, it means that the user has a large space for energy saving. The proportion of waste heat and pressure resources can reflect the recyclable waste heat and pressure resources of users. Carbon emission is also an important indicator of energy conservation and environmental protection. In the terminal energy consumption, electric energy is used to replace the energy consumption mode of scattered coal and fuel oil. The proportion of electric energy is the ratio of electric power and total energy, which can reflect the space of electric energy replacement.

The indicators of demand response service demand include transferable load capacity, reducible load capacity and interruptible load capacity.

The indicator of subjective factors is used to evaluate the enthusiasm of users to participate in power grid operation. The indicators of subjective awareness include timeliness of electricity bill settlement, illegal use of electricity, awareness of energy conservation and environmental protection, and enthusiasm of policy response.

\section{Integrated energy demand assessment model}

\subsection{Data preprocessing}

The assessment of multiple indicators refers to the summary of the information of multiple indicators of the assessment object so as to understand the advantages and disadvantages of the assessment object under a certain standard on the whole. To reflect the overall characteristic of the assessment object, it is necessary to organize multiple individual indicators. Therefore, there needs to be comprehensiveness between indicators ${ }^{[8]}$. However, due to the different nature and different units of indicator, there is often a lack of comprehensiveness between each indicator. In order to solve the problem that the indicator data can be integrated, it is necessary to conduct dimensionless processing for the indicator data.

For a certain assessment indicator, the assessment value of $\mathrm{k}$ assessment objects is composed of vectors, and dimensionless preprocessing is performed on the data:

$$
x^{\prime}=x\left(\sum_{i=1}^{k} x_{j}^{2}\right)^{-\frac{1}{2}}
$$

There are two kinds of indicators in the index system, the benefit index, which we expect to have as many values as possible, and the cost index, which we expect to have as few values as possible. Benefit index are now used as the standard type to convert cost index into very large indicators.

$$
x^{*}=\left(x^{\prime}\right)^{-1}
$$

Where, $x^{\prime}$ is the original the cost index, and $x^{*}$ is the benefit index converted from the cost index.

\subsection{First level index assessment based on AHP}

The first-level indexes lack quantitative data and need more comparative analysis, comparative judgment and comprehensive thinking among the indexes. The AHP[9] mainly relies on the experience and subjective ideas of decision-makers, so the first-level indexes use the AHP to determine their respective weights.

The main steps of AHP include constructing paired comparison matrix, calculating weight vector and checking consistency.

1) Construct paired comparison matrix

First, experts are invited to compare the five first level indexes in pairs between two indexes and construct paired comparison matrix $A$ :

$$
A=\left(a_{i j}\right), \quad a_{i j}>0, a_{i j}=1 / a_{j i}, a_{i i}=1
$$

In the formula, $a_{i j}$ is the ratio of importance among indicators, which is quantified according to the comparison scale of indicator factors. The quantitative comparison table of indicator factors is shown in Table 1.

Table 1. The quantitative comparison table of indicator factors.

\begin{tabular}{cc}
\hline scale value & Scale significance \\
\hline 1 & equally important \\
3 & weakly important \\
5 & Obviously important \\
7 & Highly important \\
9 & Absolutely Importance \\
$2,4,6,8$ & The median of the adjacent judgments above \\
\hline
\end{tabular}

2) Calculate the weight of the vector

The pairwise comparison matrix $A$ only gives the pairwise weight comparison between the indexes, but does not give the specific weight of each index. Without considering the weight influence of other indexes, the first-level index layer is arranged in a single order, and the weight vector of each index is calculated.

The geometric mean of each line element in the paired comparison matrix is calculated according to the following formula:

$$
\begin{gathered}
W_{i}=\left(\prod_{j=1}^{n} a_{i j}\right)^{\frac{1}{n}} ; i, j=1,2, \cdots, n \\
W=\left[W_{1}, W_{2}, \cdots, W_{n}\right]
\end{gathered}
$$

Vector $W$ is normalized:

$$
w_{i}=W_{i}\left(\sum_{i=1}^{n} W_{i}\right)^{-1}
$$

The weight is determined as:

$$
w=\left(w_{1}, w_{2}, \cdots w_{n}\right)
$$

3) Check the consistency

In order to evaluate the reliability of pairwise comparison matrices, consistency tests must be carried out. First, the consistency index $C I$ is calculated: 


$$
C I=\left(\lambda_{\max }-n\right)(n-1)^{-1}
$$

Where $n$ is the order of matrix $A . \lambda_{\text {max }}$ is the maximum characteristic root of matrix $A$, and:

$$
\lambda_{\max }=\sum_{i=1}^{n} \sum_{j=1}^{n} w_{j} a_{i j}\left(n w_{i}\right)^{-1}
$$

When $C I=0$, matrix $A$ has complete consistency. When $C I$ is close to 0 , matrix $A$ has satisfactory consistency. The larger the $C \mathrm{I}$, the greater the inconsistency.

Considering the consistency of the deviation may be caused by random reasons, the random consistency index $R I$ is introduced when testing if the consistency of judgment matrix is satisfied. $R I$ is related to the order of matrix $A$. In general, the greater the matrix order number, the greater the consistency in the possibility of random deviation. The consistency index $\mathrm{CI}$ is divided by the average consistency index $R I$ to obtain the consistency ratio $C R$ :

$$
C R=C I(R I)^{-1}
$$

Partial values of the average consistency index $R I$ are shown in Table 2.

Table2 standard value of the random consistency index $R I$.

Table 2. standard value of the random consistency index $R I$

\begin{tabular}{ccccccccc}
\hline $\begin{array}{c}\text { The } \\
\begin{array}{c}\text { matrix } \\
\text { order }\end{array}\end{array}$ & 1 & 2 & 3 & 4 & 5 & 6 & 7 & 8 \\
\hline RI & 0 & 0 & 0.58 & 0.89 & 1.12 & 1.26 & 1.36 & 1.41 \\
\hline
\end{tabular}

If $C R<0.1$, then the judgment matrix is considered to meet the consistency test requirements; if $C R \geq 0.1$, then the judgment matrix is considered not to meet the consistency test standards and needs to be modified.

\subsection{Second-level index assessment based on entropy weight method}

Entropy weight method is a method to determine the weight of each index according to the amount of information provided by the observed value of each index. Since the second level index has quantitative data, it does not need to rely on the subjective judgment of the decision maker.

Entropy is a measurement of the disorder of a system $^{[10]}$. If the information entropy of the index is smaller, it indicates that the greater the variation degree of the index value is, the more information it provides, and the greater the role of prayer and its weight in the comprehensive evaluation will be.

There are $n$ assessment objects and $m$ assessment indexes, and the matrix is formed from the original data:

$$
X=\left(\mathrm{x}_{i j}\right)_{m \times n}=\left[\begin{array}{cccc}
x_{11} & x_{12} & \cdots & x_{1 n} \\
x_{21} & x_{22} & \cdots & x_{2 n} \\
\vdots & \vdots & \cdots & \vdots \\
x_{m 1} & x_{m 2} & \cdots & x_{m n}
\end{array}\right]
$$

Where, $x_{i j}$ is the evaluation value of the evaluation index $i$ of the evaluation object $j$.

The entropy value of the evaluation index $i$ is calculated as follows:

$$
e_{i}=-k \sum_{j=1}^{n} x_{i j} \ln x_{i j}
$$

Where

$$
k=(\ln m)^{-1}
$$

The difference coefficient of the evaluation index $i$ is calculated as follows:

$$
h_{i}=1-e_{i}
$$

Calculate the entropy weight of the index i:

$$
q_{i}=h_{i}\left(\sum_{i=1}^{n} h_{i}\right)^{-1}
$$

The weight vector is:

$$
q=\left(q_{1}, q_{2}, \cdots q_{m}\right)
$$

\subsection{Comprehensive assessment model}

The comprehensive weighted user energy comprehensive evaluation model is as follows:

$$
r=\sum_{i=1}^{n} w_{i}\left(\sum_{j=1}^{m} q_{i j} x_{i j}\right)
$$

\section{4 case study}

Fifty industrial users were evaluated and clustered according to the first level index vector obtained by the evaluation. The fifty users were divided into four clusters by $K$-means clustering algorithm[11]. Then the average index scores of the four clusters of users were compared to analyse the energy-use characteristics of all kinds of users. The first level index assessment results of each clusters are shown in figure3, and the final integrated assessment results are shown in figure4.

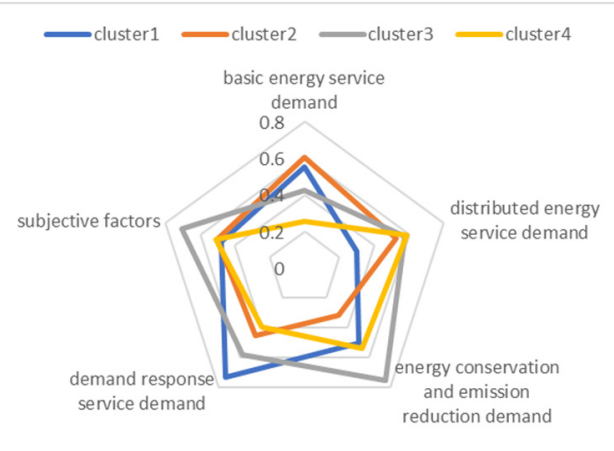

Figure 3.The first level index assessment results 


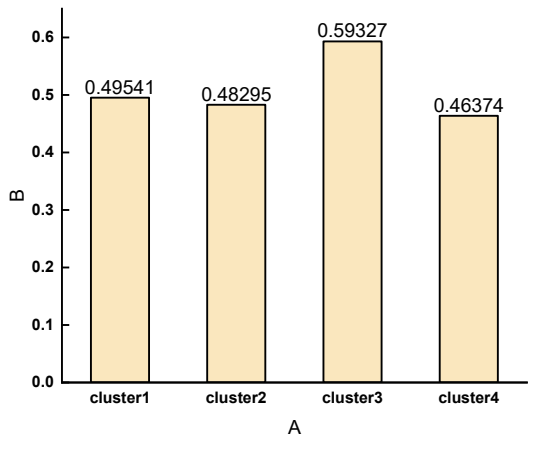

Figure 4. The final integrated assessment results

From Figure 2, the demand for basic energy services in cluster 1 is relatively high, while the demand for distributed energy services and the demand for energy conservation and emission reduction are relatively low. The demand response service demand is the highest among the four clusters, and the subjective factors score is medium. The conclusion is that it needs more basic energy services. For cluster 2, the demand for basic energy services is the highest among the four clusters, and the demand for distributed energy services is also high. The demand response service demand and the demand for energy conservation and emission reduction are low, and the subjective factor score is medium, indicating that it needs more basic energy services and distributed energy services. Cluster 3 energy service demand as the basis of the medium. Distributed energy service demand, demand response service demand, demand for energy conservation and emissions reduction and subjective factors score are also high. The comprehensive score is the highest in four clusters, which means that Cluster 3 is one of the most important aspects of the comprehensive energy service customers. Cluster4 has a high demand fordistributed energy services and demand response services. Other demands are low. The comprehensive score is low. So, they are not the key service objects of comprehensive energy services.

\section{Conclusion}

The integrated energy service demand evaluation indexes proposed in this paper can effectively reflect the demand of users for integrated energy service. By means of AHP and entropy weight method, the index can be weighted, which can reflect the demand of users quantitatively. By clustering the first level indexes, it can help the integrated energy service company to distinguish and select key customers.

\section{Acknowledgments}

This work was supported by Institute of Economy and Technology, State Grid Anhui Electric Power Co., Ltd. (No. B3440818K005).

\section{References}

1. Wang Z, Zhu Y, Zhu Y, et al. Energy structure change and carbon emission trends in China[J]. Energy, 2016, 115:369-377.

2. Sugihara H , Komoto J , Tsuji K . A Multi-objective Optimization Model for Determining Urban Energy Systems under Integrated Energy Service in a Specific Area[J]. Electrical Engineering in Japan, 2010, 147(3):20-31.

3. Yiqing L, Zuogang G, Lei Y U, et al. Competition Strategy of Integrated Energy Service Provider Under Pool-Based Market Mechanism[J]. southern power system technology, 2019.

4. WANG Jingwen, LI Huaqiang, LI Xuxiang, et al.Utility Model and Demand Assessment Method of Integrated Energy Service [J]. Proceedings of the CSEE, 2020, 40(2).

5. Jia Hongjie, Wang Dan, Xu Xiandong, et al . Researchon some key problems related to integrated energysystems $[\mathrm{J}]$. Automation of Electric Power Systems, 2015, 39(7) : 198207(in Chinese).

6. Huang Hanqi, Mao Chengxiong, Wang Dan, et al. Modeling summarizing of distributed renewable energy power generation system[J]. Proceedings of the Chinese Society of Universities , 2010 , 22(5) : 1-18, 24(in Chinese).

7. Zhang Dongxia, Miao Xin, Liu Liping, et al. Research on development strategy for smart grid big data[J]. Proceedings of the CSEE, 2015, 35(1): 2-12(in Chinese).

8. Forman E H , Gass S I . The Analytic Hierarchy Process--An Exposition[J]. Operations Research, 2001, 49(4):469-486.

9. Delgado A , Romero I . Environmental conflict analysis using an integrated grey clustering and entropy-weight method: A case study of a mining project in Peru[J]. Environmental Modelling \& Software, 2016, 77(Mar.):108-121.

10. Christos Bouras, Vassilis Tsogkas. W-kmeans: Clustering News Articles Using WordNet[M]// Knowledge-Based and Intelligent Information and Engineering Systems. Springer Berlin Heidelberg, 2010. 